\title{
Dynamic wettability and contact angles of poly(vinylidene fluoride) nanofiber membranes grafted with acrylic acid
}

\author{
F. L. Huang 1 , Q. Q. Wang 1 , Q. F. Wei ${ }^{1 *}$, W. D. Gao ${ }^{1}$, H. Y. Shou ${ }^{2}$, S. D. Jiang 1 \\ ${ }^{1}$ Key Laboratory of Eco-Textiles(Jiangnan University), Ministry of Education, 214122 Wuxi, China \\ 2Zhejiang Province New Textile Research \& Development Emphasised Laboratory, Zhejiang Textile \& Garment Science \\ \& Technology Co., Ltd., 310009 Hangzhou, China
}

Received 18 March 2010; accepted in revised form 20 May 2010

\begin{abstract}
Wettability has been recognized as one of the most important properties of fibrous materials for both fundamental and practical applications. In this study, the plasma induced grafting of acrylic acid (AAc) was applied to improve the wettability of the electrospun poly(vinylidene fluoride) (PVDF) nanofiber membranes. The diameter and chemical structure of the modified PVDF nanofibers were characterized by scanning electron microscopy (SEM) and Fourier transform infrared (FTIR). Nitrogen adsorption based on BET (Brunauer, Emmett and Teller) principle was employed to measure the specific surface areas and porosities of the modified nanofiber membrances. The contact angles of the modified membrane were evaluated by drop shape analysis (DSA) and the modified Washburn method. The dependence of contact angles on specific surface area and porosity was also discussed in this paper. Water adsorptions were used to evaluate the dynamic wetting behavior of the grafted membranes by a dynamic adsorption apparatus (CDCA100-F). The experimental results revealed that the wettablity of the modified PVDF membrane was significantly affected by both surface and porous contact angles.
\end{abstract}

Keywords: polymer membranes, electrospinning, contact angles, poly (vinylidene fluoride)

\section{Introduction}

PVDF has been intensively studied due to its excellent bulk properties, such as high electric resistance as well as good thermostability, light weight and good processability [1-3]. These properties have made PVDF increasingly used in various fields such as filtration, air cleaning, and rechargeable batteries. These applications require materials with well-defined properties and functionalities. Nanofibers produced by electrospinning have attracted a great deal of attentions in these applications due to its remarkable properties, such as small fiber diameters, porous structures as well as high surface area $[4,5]$. However, their applications are hampered in many cases because of its poor wettability and adhesion property with other materials [6].
In recent years, various techniques have been tried to improve the wettability of PVDF materials, such as plasma treatment, iron irradiation and sputter coating $[6,7]$. In these modifications, plasmainduced graft polymerization of vinyl monomers has been found to be an extremely attractive technique for chemically modifying the surfaces of polymeric materials [8]. Although both surface energy and surface roughness are the dominant factors for wettability of materials, surface roughness is the key factor once the components of materials have been selected.

In this study, electrospun PVDF nanofiber membranes were modified by plasma-induced grafting of acrylic acid to improve their wettablity. The PVDF membranes with different structures were 
prepared by electrospinning for the investigation into the relationship between dynamic wettablity and fibrous structures.

The wettability of a material can be characterized by contact angles. Two types of measurements, drop shape analysis and Washburn method [9], have been widely used to characterize the contact angles. However, due to the complicated surface structures, few literatures have focused on the surface contact angle of nanofiber membrane. And also, the complicated internal geometry in the nanofiber membrane has also been the obstructive factor for the analysis of Washburn contact angle. In this work, the Washburn equation was modified to evaluate the porous contact angles of the grafted PVDF nanofiber membrane, and the effect of porous structures on contact angles was also discussed.

\section{Experimental}

\subsection{Materials}

PVDF with average molecular weight $\left(M_{n}\right)$ of $5.5 \cdot 10^{5} \mathrm{~g} \cdot \mathrm{mol}^{-1}$ was purchased from Shanghai $3 \mathrm{~F}$ New Materials Co., Ltd (Shanghai, China). N,NDimethyl formamide (DMF), acetone and acrylic acid (AAc) were supplied by Sinopharm Chemical Reagent Co., Ltd (Shanghai, China) and used without further purification.

\subsection{Preparation of PVDF nanofibers}

PVDF nanofiber membranes with four different fiber diameters were prepared in this study. PVDF was dissolved in a mixture of DMF and acetone $(20 / 80, w / w)$ to make $15 \%$ (wt) solutions for electrospinning.

The apparatus for electrospinning included a plastic syringe, an 18 gauge stainless steel needle, a microinfusion pump (Medical Instrument Co., Zhejiang, China), a high-voltage power supply (Dongwen Co., Tianjing, China), and an aluminum foil as the fiber collector. The ejection rate of the solution was set at $1.0 \mathrm{ml} / \mathrm{h}$, and the distance between the needle tip and the fiber collector was $15.0 \mathrm{~cm}$. Electrospinning was performed at the electrostatic voltages of $8,12,16$ and $20 \mathrm{kV}$ respectively with the adjustable-voltage power supply. The electrospinning was performed in a fume chamber at the temperature of $20^{\circ} \mathrm{C}$ and humidity of $45 \%$. After three hours deposition, all the membranes were collected on the aluminium foil substrate. The membranes were dried in vacuum to remove the solvent. The membranes were then cut at the weight of $5.0 \mathrm{~g}$.

\subsection{Plasma-induced grafting of PVDF nanofibers}

The process of plasma pretreatment followed by surface grafting with acrylic acid (AAc) on the membrane is described in Figure 1. The nanofiber membrane was treated with an argon plasma which was performed in a HD-1A vertical plasma treatment machine (Changzhou Shitai Co, China). The pretreatment was performed at a pressure of $15 \mathrm{~Pa}$ and power of $75 \mathrm{~W}$ for $120 \mathrm{~s}$. After being taken out from the reactor, the sample was allowed to stay in air atmosphere for $30 \mathrm{~min}$ and then immersed in a solution of acrylic acid (30\%,wt). The grafting reaction was carried out by placing ampoules at $60^{\circ} \mathrm{C}$ temperature in a water bath for $2 \mathrm{~h}$. The samples were, then, washed extensively in deionized water at $60^{\circ} \mathrm{C}$ in a water bath to remove the poly (acrylic acid) which was not covalently bound to the PVDF surface. The membranes were then dried in an oven at $70^{\circ} \mathrm{C}$.

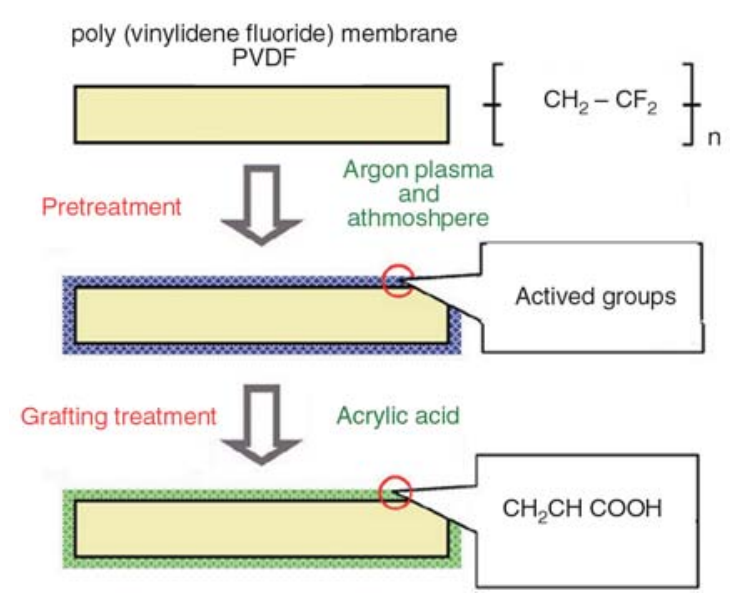

Figure 1. Schematic diagram of pretreatment and grafting on PVDF nanfiber membrane

\subsection{SEM and FTIR}

The fibrous structures of the nanofibers were examined using a scanning electron microscopy (SEM, JEOL JSM-5610LV, Japan). Before SEM imaging, 
the samples were sputtered with a thin layer of gold. The diameters of nanofibers were also measured using the software Photoshop 10.0.

The surface chemistry of the modified PVDF nanofiber membranes was examined by Fourier Transform Infrared (FTIR) (Thermo Electron Corporation). Spectra were recorded in air by use of a FTIR Nexus spectrometer. Approximately 64 scans were co-added to achieve the signal-to-noise ratio shown. The spectral resolution was $4 \mathrm{~cm}^{-1}$.

\subsection{Specific surface area and pore structure}

The specific surface areas and pore structures of the grafted nanofiber membranes were examined using low temperature $(77.38 \mathrm{~K})$ nitrogen adsorption isotherms measured over a wide range of relative pressure from 0.02 to 1 . Adsorption measurements were performed on an ASAP2010 volumetric adsorption apparatus. High purity nitrogen (99.9999\%) was used in this experiment. Prior to measurement, the samples were degassed at $100^{\circ} \mathrm{C}$ for $3 \mathrm{~h}$ in the degas pot of the adsorption analyzer.

\subsection{Contact angles}

The surface contact angles were firstly measured on a Drop Shape Analysis System (DSA100) (KRUSS, Germany). Deionized water was dropped onto the sample from a needle on a microsyringe during the test. A picture of the drop was captured after the drop set onto the sample. The contact angles could be calculated by the software through analyzing the shape of the drop. The contact angle $\theta$ was an average of 20 measurements.

Washburn method [9] has been recognized as the most effective tool to analyze the contact angle of powder materials. In this study, modified Washburn method was used to characterize the contact angles of porous media. The measurement was performed using a CDCA-100F (a dynamic contact angle apparatus made by the Camtel Ltd. Co., UK). The method for measuring the contact angle relies on the test fluids penetrating the porous sample, which can be expressed by the theory of Washburn (Equation (1)):

$$
\frac{l^{2}}{t}=\frac{\sigma r \cos \theta}{2 \eta}
$$

Where $l, \sigma, r, \theta$ and $\eta$ represent rising height, surface tension of liquid, radius of capillary, advancing contact angle and viscosity of liquid, respectively.

For the nanofiber membrane, this equation can be modified such that they are seen as a bundle of capillaries with a mean radius of capillary, $r$. A modified Washburn equation can be used (Equation (2)):

$$
\frac{l^{2}}{t}=\frac{(c r) \sigma \cos \theta}{2 \eta}
$$

In this equation, $c$ is a constant to estimate the tortuous path of the capillaries.

The relationship becomes non-linear at higher $\sigma$ values i.e. the rise height decreases. Once $(c r)$ is determined for a given sample, the advancing angle of liquids with $\cos \theta<1$. $l$ can be calculated by replacing the rise height of the liquid with the weight gain of the sample. Hence the Equation (2) becomes Equation (3):

$$
\frac{W^{2}}{t}=\frac{\left[(c r) \omega^{2}\left(\pi R^{2}\right)\right] \rho^{2} \sigma \cos \theta}{2 \eta}=\frac{K \rho^{2} \sigma \cos \theta}{2 \eta}
$$

Where $W, \rho, \omega, R$, represent weight of penetrating liquid, density of measuring liquid, relative porosity and inner radius of the measuring tube.

For a given sample, i.e. nanofiber membrane in this study, $K$, the geometric factor was found by conducting a preliminary test on the nanofiber membrane using n-hexane as a totally wetting liquid $(\cos \theta=1)$, which can be characterized by the Equation (4):

$$
K=\frac{2 W^{2} \eta}{t \rho^{2} \sigma}
$$

Thus, the Washburn contact angle of nanofiber membrane can be calculated by Equations (3) and (4).

\subsection{Dynamic wetting}

Dynamic wetting tests were performed on a Camtel CDCA-100F dynamic adsorption apparatus (Camtel, UK). Each sample was cut to a size of $1 \mathrm{~cm} \times$ $5 \mathrm{~cm}$ with sharp scissors. When the specimen was immersed into water, the weight of adsorbed water was detected and recorded. The dynamic water adsorption was plotted as a function of time. 


\section{Results and discussion \\ 3.1. Diameters of PVDF nanofbers}

To investigate the effect of fibrous structures on contact angle and wettability, the nanofibers with various diameters were prepared by adjusting electrospinning voltage. SEM images of the grafted membranes prepared by electrospinning with different voltages are presented in Figure 2. It can be clearly observed form the images that the average diameter of the PVDF nanofibers decreases with increasing applied voltage. The effect of voltage on diameters of nanofibers has been extensively investigated in some literatures [10-12]. The drawing rate of the nanofibers can be enhanced by increasing the electrostatic forces brought by the electrostatic voltage. The average diameters of the grafted PVDF nanofibers electrospun with different static voltages are illustrated in Figure 2f.

\subsection{Surface chemistry}

Figure 3 shows the infrared spectra for the original PVDF nanofiber membrane, the samples treated with argon plasma and acrylic acid grafting. The spectrum of original PVDF membrane (Figure 3a) exhibits some intensive bands at 1400, 1280, 1076, $835 \mathrm{~cm}^{-1}$, which are assigned to the characteristic vibration of $\mathrm{C}-\mathrm{H}$ and $\mathrm{C}-\mathrm{F}$. Compared to the pris-

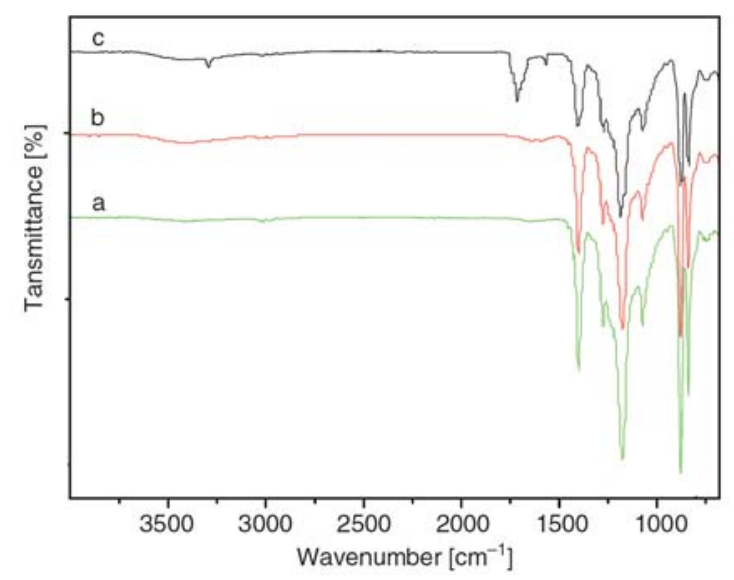

Figure 3. FTIR spectrum of (a) original PVDF membrane, (b) plasma treated PVDF membrane and (c) grafted PVDF membrane

tine PVDF membrane, there is no obvious change on the membrane after plasma treatment as illustrated in Figure 3b. This step only attributes to the formation of activated radicals. For grafted nanofiber membrane, the appearance of representative bands at $3380 \mathrm{~cm}^{-1}$, as shown in Figure 3c, which attests the presence of the $-\mathrm{O}-\mathrm{H}$. From the spectra, stretching vibration of $\mathrm{O}=\mathrm{C}-\mathrm{O}-$ at $1727 \mathrm{~cm}^{-1}$ is well observed as well as the symmetric stretching vibration of $-\mathrm{C}=\mathrm{O}$ at $1695 \mathrm{~cm}^{-1}$. It can be concluded from these observations that a layer of acrylic acid is grafted on the PVDF nanofiber membrane. This result can be also ascertained from the

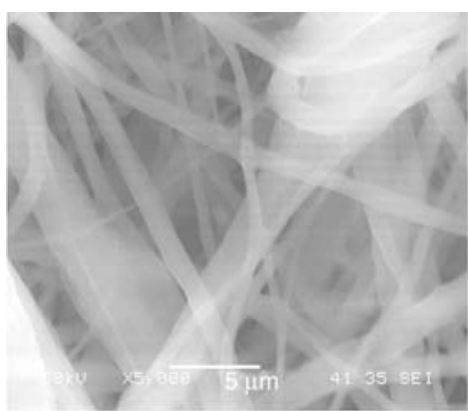

a)

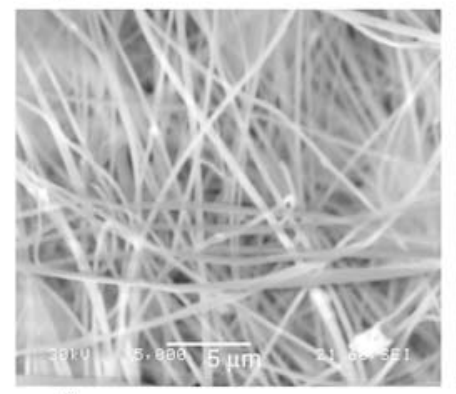

d)

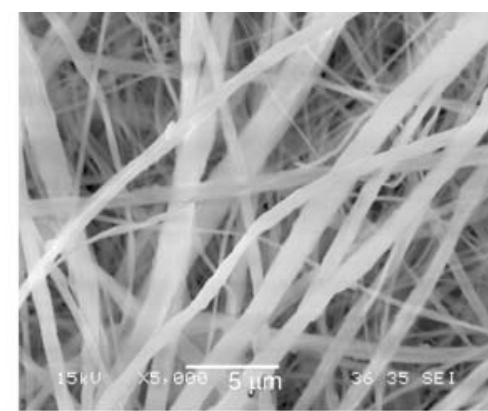

b)

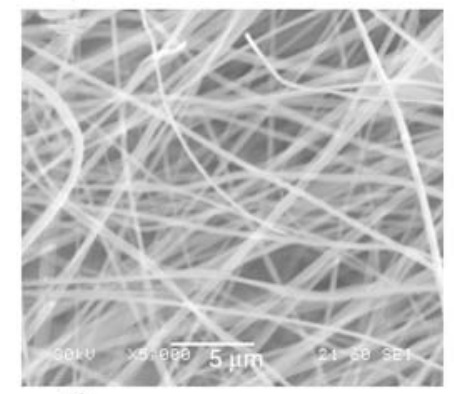

e)

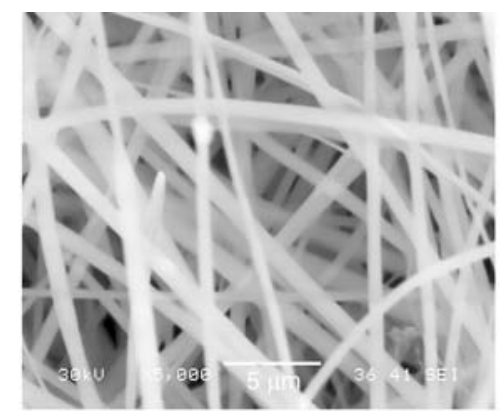

c)

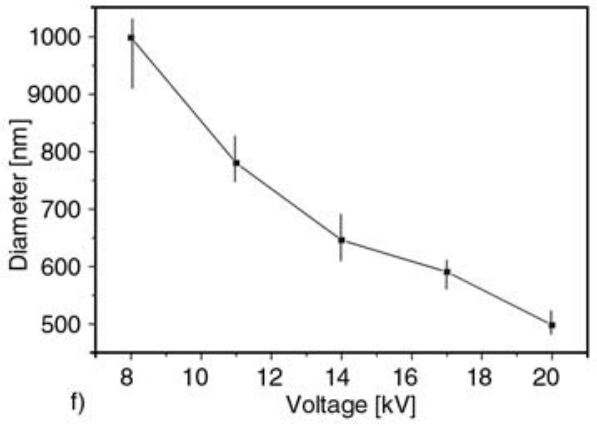

Figure 2. SEM images of PVDF membranes prepared with voltage of (a) $8 \mathrm{kV}$, (b) $11 \mathrm{kV}$, (c) $14 \mathrm{kV}$, (d) $17 \mathrm{kV}$, (e) $20 \mathrm{kV}$ and (f) curve of diameter with voltage 
typical vibration of $\mathrm{C}-\mathrm{F}$ and $\mathrm{C}-\mathrm{H}$ from 800 $1400 \mathrm{~cm}^{-1}$, which obviously appears weakened on the FTIR spectra of modified membrane.

\subsection{Specific surface area and porous structure}

It is well known that the difference in fiber size leads to change in the material structures such as specific surface area and pore size distribution. In this study, electrospun voltage was adjusted to control fibrous structures of the modified PVDF membrane. The BET specific surface areas of the nanofiber mats grafted with acrylic acid are summarized in Figure 4. It can be seen from Figure 4 that BET specific surface area has a tendency to increase with reduction of fibre diameter obtained by reducing the voltage. The specific surface area increases from 8 to $24 \mathrm{~m}^{2} / \mathrm{g}$ as the diameter reduced from 1000 to $500 \mathrm{~nm}$ (and voltage raised from 8 to $22 \mathrm{kV}$ ). This phenomenon is attributed to the enhanced stretching effect of the electrostatic forces as the electrostatic voltage increases. Each value of BET surface area is much higher than the traditional fibrous materials, though these values are slightly lower than that of untreated nanofiber membrane. The specific surface area of untreated nanofiber membrane electrospun with the voltage of $22 \mathrm{kV}$ is about $28.4 \mathrm{~m}^{2} / \mathrm{g}$.

A shifting trend in the porosity of the grafted PVDF membrane is also revealed in Figure 4 and the results indicate that the porosity doesn't show a monotone increase in contrast to BET surface area. It is shown that the porosity of the grafted PVDF membrane changes in the range between 25.1 and $59 \%$. As the applied voltage alters from 8 to $14 \mathrm{kV}$,

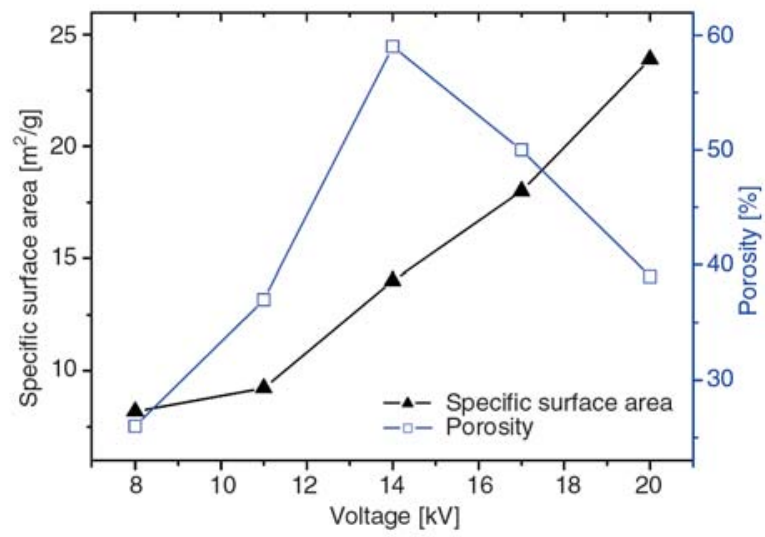

Figure 4. Specific surface area and porosity of PVDF nanofiber membrane the porosity presents an evident increase and reaches to a top value of 59\%. However, the values of the porosity have an opposite trend as the voltage is above $14 \mathrm{kV}$. The porosity of the PVDF nanofiber membrane decreases from $59 \%(14 \mathrm{kV})$ to $38.8 \%(20 \mathrm{kV})$. This phenomenon may be attributed to a more compressed assembly of the nanofibers generated by the higher voltage.

\subsection{Contact angles}

Surface contact angle of the grafted nanofiber membrane is measured using sessile drop observation. Figure 5a shows a water droplet formed on the untreated PVDF nanofiber membrane. The surface contact angle of the nanofiber membrane is $91.2^{\circ}$, in agreement with the strong hydrophobicity of PVDF material to water. The image in Figure 5b shows a significant decrease in the contact angle on the grafted PVDF membrane, which may be ascribed to the grafting of hydrophilic radical, $-\mathrm{COOH}$. However, it is proved from the observations that the surface chemical feature is not the only factor influencing the surface contact angle. The membranes obtained with different electrospinning voltages and grafted under the same conditions present varied contact angles, as illustrated in Figure 6. This observation can be explained by

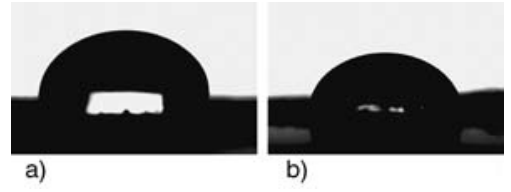

Figure 5. Micrograph of surface contact angle of (a) untreated membrane (b) membrane grafted with acrylic acid

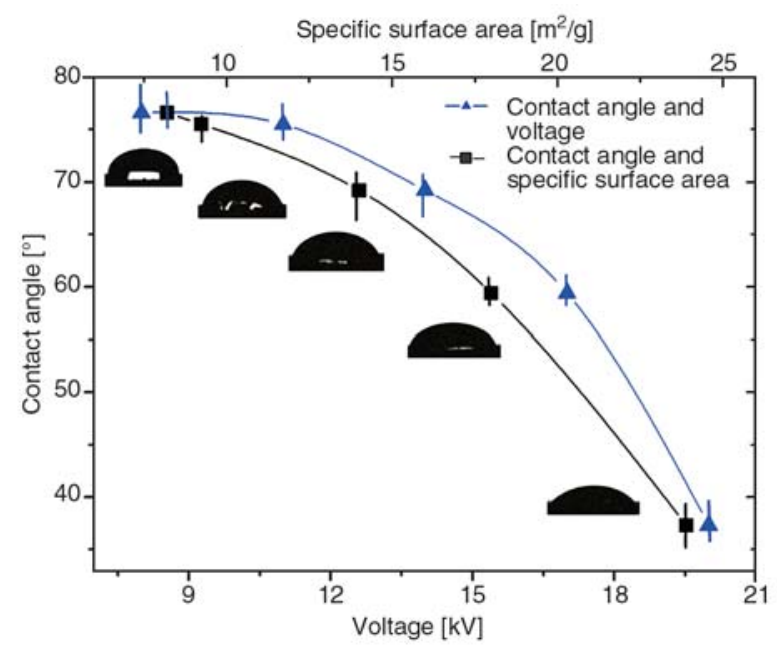

Figure 6. Surface contact angles of the PVDF membrane 
the Wenzel equation [13]. This equation indicates that the water contact angle of the surface decreases with increasing surface roughness when the surface is composed of hydrophilic substances. Roughness, however, is so complicated that it is difficult to develop a general method for the roughness measurement. In this study, specific surface area based on BET method is used for roughness characterization (see Equations (5) and (6)):

$$
\begin{aligned}
& B=\frac{S_{r}}{m} \\
& R=\frac{S_{r}}{S_{p}}=\frac{m B}{S_{p}}
\end{aligned}
$$

Where $S_{r}$ is the real surface area of nanofibrous membrane, $S_{p}$ is the projected area, $B$ is the specific surface area, and $m$ represents the mass of a membrane.

Then the contact angle of the grafted membrane can be expressed by the Equations (7) and (8):

$$
\begin{aligned}
& \cos \theta^{\prime}=R \cos \theta=\frac{m B}{S_{p}} \cos \theta \\
& \theta^{\prime}=\arccos (R \cos \theta)=\arccos \left(\frac{m B}{S_{p}} \cdot \cos \theta\right)
\end{aligned}
$$

It can be seen from the Equation (8) that the contact angle, $\theta^{\prime}$ of the membrane depends on the ideal contact angle, $\theta$ and surface area, and the specific surface area plays an important role in the contact angle of a material. Larger specific surface area, as shown in Figure 6, leads to lower contact angles, fitting the curve of arccosine.

Porous contact angle is an important fact influencing the wetting behavior of fibrous membrane, which can be calculated by a modified Washburn equation as described in Section 2.3. As displayed in Figure 7, the Washburn contact angles of the five grafted membranes were calculated to be 67,50 , 40,46 and $44^{\circ}$, respectively. The values obtained by this technique show an obvious difference from those obtained by sessile drop tests. The contact angle doesn't show a monotone change with the electrospinning voltage. Interestingly, it is also found from Figure 7 that the Washburn contact angle of the PVDF nanofiber membrane is closely related to its porosity. The membrane with higher porosity shows a lower contact angle. The highest porosity of $59 \%$ seems to bring the lowest contact

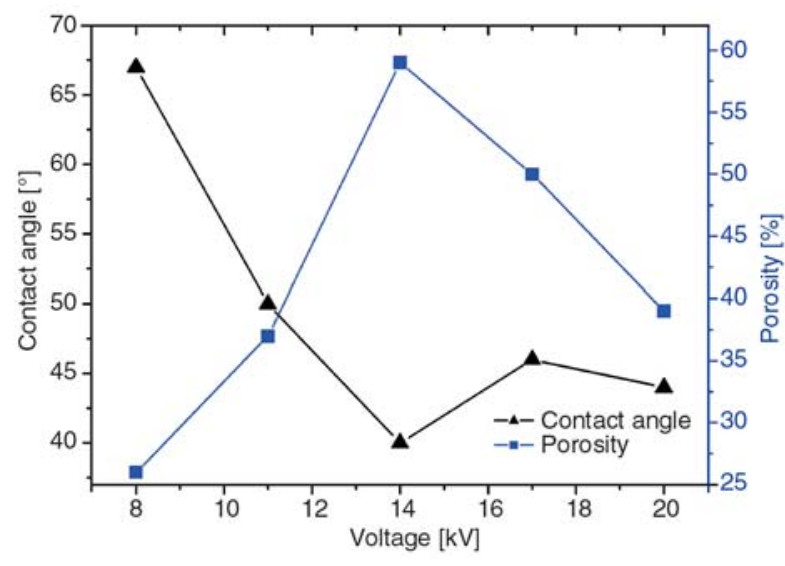

Figure 7. Diagram of the Washburn contact angle and porosity

angle of $40^{\circ}$. This phenomenon can be also analyzed by the modified Washburn Equation (3). However, it should also be noted that the Washburn contact angle of the membrane presents a descendent trend when the electrospinning voltage is over $17 \mathrm{kV}$, which may be attributed to the effect of other porous factors in the nanofiber membrane, for example, pore size and its distribution.

\subsection{Dynamic wetting}

The results of the water adsorption tests reveal the dynamic wetting behavior of the PVDF nanofiber membranes. The membrane shows a very low adsorption before the acrylic acid grafting, as shown in Figure 8. When the sample is immersed into water, the effect of buoyancy of water pushes the materials upwards since the surface contact angle is higher than $90^{\circ}$. This observation confirms the hydrophobic behavior of the PVDF nanofiber membrane. It can be seen from Figure 8 that the adsorption curve of the grafted membrane (electro-

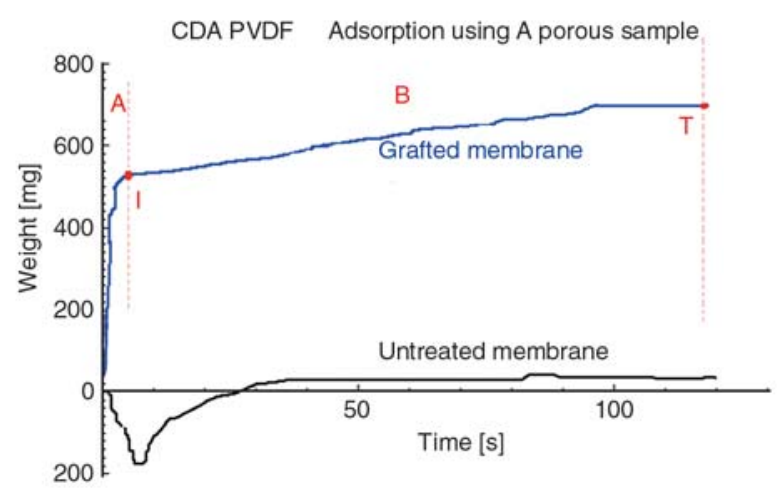

Figure 8. Dynamic water adsorption of PVDF membrane before and after acrylic acid grafting 
spun with voltage of $20 \mathrm{kV}$ ) displays a contrary evolution to that of the untreated membrane. The acrylic acid grafting considerably improves the water adsorption properties of the PVDF nanofiber membrane due to the lower contact angles.

The effect of the electrospinning voltage on the water wetting behavior can be obviously observed from the dynamic adsorption tests, as illustrated in Figure 9. All five samples electrospun with different voltages were grafted with acrylic acid under the same processing conditions. It can be seen from the image that all the grafted membranes show hydrophilic properties and the amount of water adsorbed is all over $300 \mathrm{mg}$. Although the adsorption curves show similar trends, they also exhibit varied phenomena as indicated in Figure 9.

It is observed from the adsorption curves that they appear to have two zones, as shown in Figure 9. The first zone, $A$ is the initial water adsorption, which occurs over the first few seconds. And a crucial inflexion labeled $I$ represents the top value of water adsorption in the first zone. The weight of adsorption water of each sample can be respectively denoted by $W_{8}, W_{11}, W_{14} W_{17}, W_{20}$, corresponding to voltage of $8,11,14,17$ and $20 \mathrm{kV}$, and the time of reaching to each inflexions is $T_{8}, T_{11}$, $T_{14}, T_{17}$ and $T_{20}$. These values can be easily obtained from Figure 9, which are expressed by the following orders: $T_{8}>T_{11}>T_{14}>T_{17}>T_{20}$ and $W_{8}<W_{11}<W_{14}<W_{17}<W_{20}$. During the initial adsorption, it can be found that higher voltage results in a quicker adsorption and a larger amount of water absorbed. Since the water mainly contact with the outmost fibers in the first zone, the surface contact angle may play the essential role in the ini-

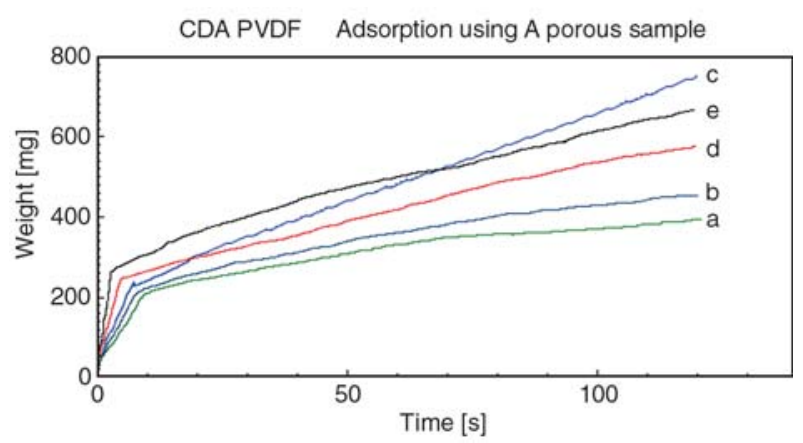

Figure 9. Dynamic water adsorption of the membrane electrospun with (a) $8 \mathrm{kV}$ (b) $11 \mathrm{kV}$ (c) $14 \mathrm{kV}$ (d) $17 \mathrm{kV}$ and (e) $20 \mathrm{kV}$ tial adsorption. The lower contact angle leads to the quicker adsorption and larger weight of water.

Then, the water is adsorbed into the fibrous webs and transferred through capillary. This could be called transferring zone, $B$, as shown in Figure 9. It can be seen from the image that another important point, $T$, which presents the total water adsorption. It is observed from Figure 9, that the grafted membrane with the electrospinning voltage of $14 \mathrm{kV}$ obtained the largest weight of adsorption. It may be explained by the effect of porous contact angle on the water adsorption. In the transferred zone, lower porous contact angle leads to faster transferring rate and a larger amount of adsorption. And it can also be concluded from the results that surface contact angle determines the initial adsorption, and the porous contact angle influences the capillary adsorption.

\section{Conclusions}

This study has revealed that the plasma-induced grafting of acrylic acid significantly improved the wetting behaviour of the PVDF nanofiber membrane. PVDF membranes with different surface structures were prepared by electrospinning to investigate the relationship between dynamic wetting behavior and structures. The BET tests have shown that applied voltage obviously altered the specific surface area and porosity, which have been proved to be the key facts influencing the surface and Washburn contact angles. It is observed from the adsorption curves that they appear to have two zones, initial water adsorption and capillary water adsorption. It was also found from the water adsorption measurement that surface contact angle determined the initial adsorption, and the porous contact angle influenced the capillary adsorption.

\section{Acknowledgements}

The work was financially supported by the Fundamental Research Funds for the Central Universities of Jiangnan University (No. JUSRP20903), the Program for Key Laboratory of Textile Science \& Technology, Ministry of Education, Donghua University, and the Doctoral Program of Higher Education of China (No. 200802951011 and No. 20090093110004) 


\section{References}

[1] Khayet M., Feng C. Y., Khulbe K. C., Matsuura T.: Preparation and characterization of polyvinylidene fluoride hollow fiber membranes for ultrafiltration. Polymer, 43, 3879-3890 (2002).

DOI: $10.1016 / \mathrm{S} 0032-3861(02) 00237-9$

[2] García-Payo M. C., Essalhi M., Khayet M: Effects of PVDF-HFP concentration on membrane distillation performance and structural morphology of hollow fiber membranes. Journal of Membrane Science, 347, 209-219 (2010).

DOI: $\underline{10.1016 / j . m e m s c i .2009 .10 .026}$

[3] Kim J-U., Park S-H., Choi H-J., Lee W-K., Lee J-K., Kim M-R.: Effect of electrolyte in electrospun poly(vinylidene fluoride-co-hexafluoropropylene) nanofibers on dye-sensitized solar cells. Solar Energy Materials and Solar Cells, 93, 803-807 (2009).

DOI: $10.1016 /$ j.solmat.2008.09.045

[4] Gao K., Hu X. G., Dai C. S., Yi T. F.: Crystal structures of electrospun PVDF membranes and its separator application for rechargeable lithium metal cells. Materials Science and Engineering: B, 131, 100-115 (2006).

DOI: $10.1016 /$ j.mseb.2006.03.035

[5] Huang Z-M., Zheng Y-Z., Kotaki M., Ramakrishna S.: A review on polymer nanofibers by electrospinning and their applications in nanocomposites. Composites Science and Technology, 63, 2223-2253 (2003). DOI: $10.1016 / \mathrm{S} 0266-3538(03) 00178-7$

[6] Hietala S., Skou E., Sundholm F.: Gas permeation properties of radiation grafted and sulfonated poly(vinylidene fluoride) membranes. Polymer, 40, 55675573 (1999).

DOI: $10.1016 / \mathrm{S} 0032-3861(98) 00768-\mathrm{X}$
[7] Liu F., Du C-H., Zhu B-K., Xu Y-Y.: Surface immobilization of polymer brushes onto porous poly(vinylidene fluoride) membrane by electron beam to improve the hydrophilicity and fouling resistance. Polymer, 48 , 2910-2918 (2007).

DOI: $\underline{10.1016 / j . p o l y m e r .2007 .03 .033}$

[8] Lu W-L., Huang C-Y., Roan M-L.: Effect of plasma treatment on the degree of AAm grafting for high-density polyethylene. Surface and Coatings Technology, 172, 251-261 (2003).

DOI: 10.1016/S0257-8972(03)00337-2

[9] Washburn E. W.: Dynamics of capillary flow. Physical Review, 17, 273-283 (1921).

[10] Helgeson M. E., Wagner N. J.: A correlation for the diameter of electrospun polymer nanofibers. AIChE Journal, 53, 51-55 (2007).

DOI: $10.1002 /$ aic. 11056

[11] Feng J. J.: Stretching of a straight electrically charged viscoelastic jet. Journal of non-Newtonian Fluid Mechanics, 116, 55-70 (2003). DOI: $10.1016 / \mathrm{S} 0377-0257(03) 00173-3$

[12] Spivak A. F., Dzenis Y. A., Reneker D. H.: A model of steady state jet in the electrospinning process. Mechanics Research Communications, 27, 37-42 (2000). DOI: 10.1016/S0093-6413(00)00060-4

[13] Wenzel R. N.: Resistance of solid surfaces to wetting by water. Industrial and Engineering Chemistry, 28, 988-994 (1936).

DOI: 10.1021/ie50320a024 